\title{
Occlusal force, electromyographic activity of masticatory muscles and mandibular flexure of subjects with different facial types
}

\author{
William CUSTODIO', Simone Guimarães Farias GOMES'1, Fernanda FAOT², Renata Cunha Matheus Rodrigues \\ GARCIA $^{3}$, Altair Antoninha DEL BEL CURY3

\begin{abstract}
1- MSc, Graduate student, Department of Prosthodontics and Periodontology, Piracicaba Dental School, State University of Campinas, Piracicaba, SP, Brazil. 2- DDS, MSc, PhD, Associate Professor, Department of Dental Prosthodontics, Dentistry School, Federal University of Pelotas, Pelotas, RS, Brazil.

3- DDS, MSc, PhD, Professor, Department of Prosthodontics and Periodontology, Piracicaba Dental School, State University of Campinas, Piracicaba, SP, Brazil.
\end{abstract}

Corresponding address: Dr. Altair Antoninha Del Bel Cury - Faculdade de Odontologia de Piracicaba - UNICAMP - Departamento de Prótese e Periodontia Av. Limeira, n 901 - Piracicaba, SP - Brasil - 13414-903 - Phone: + 55-19-2106-5294 - Fax: + 55-19-2106-5211 - e-mail: altcury@fop.unicamp.br

Received: September 17, 2009 - Modification: March 15, 2010 - Accepted: March 6, 2010

\section{ABSTRACT}

\begin{abstract}
$\mathrm{O}$ bjective: The aim of this study was to evaluate whether vertical facial patterns influence maximal occlusal force (MOF), masticatory muscle electromyographic (EMG) activity, and medial mandibular flexure (MMF). Material and Methods: Seventy-eight dentate subjects were divided into 3 groups by Ricketts's analysis: brachyfacial, mesofacial and dolychofacial. Maximum occlusal force in the molar region was bilaterally measured with a force transducer. The electromyographic activities of the masseter and anterior temporal muscles were recorded during maximal voluntary clenching. Medial mandibular flexure was calculated by subtracting the intermolar distance of maximum opening or protrusion from the distance in the rest position. The data were analyzed using ANOVA followed by Tukey's HSD test. The significance level was set at $5 \%$. Results: Data on maximum occlusal force showed that shorter faces had higher occlusal forces $(P<0.0001)$. Brachyfacial subjects presented higher levels of masseter electromyographic activity and medial mandibular flexure, followed by the mesofacial and dolychofacial groups. Additionally, dolychofacial subjects showed significantly lower electromyographic temporalis activities $(P<0.05)$. Conclusion: Within the limitations of the study, it may be concluded that maximum occlusal force, masticatory muscle activity and medial mandibular flexure were influenced by the vertical facial pattern.
\end{abstract}

Key words: Vertical dimension. Bite force. Electromyography. Masticatory muscles. Mandible.

\section{INTRODUCTION}

A variety of craniofacial morphologic features contribute to the determination of vertical facial types in humans ${ }^{19}$. However these facial characteristics can hamper masticatory system functioning ${ }^{20}$. Aside from esthetic considerations, presumed functional muscular activities are frequently mentioned as a cause of misfit or failure in prostheses ${ }^{7}$ because muscle actions induce mandibular flexure around the symphysis during opening and protrusion movements ${ }^{1,5}$. Different craniofacial morphologies can lead to differences in neuromuscular activities $8,24,26$ and in muscle cross-sectional area, volume and orientation ${ }^{4}$. Previous studies assessing the relationship between mandibular muscles and facial morphology showed that subjects with wider transverse craniofacial dimensions have thicker and consequently stronger mandibular muscles ${ }^{8}$ and present anatomic features such as small gonial angles ${ }^{25}$ and reduced lower anterior facial height ${ }^{15}$. In contrast, dolichofacial subjects present weaker muscles than do mesofacial and brachyfacial subjects ${ }^{15}$.

Occlusal forces and masticatory muscle electromyographic (EMG) activity during function are also related to vertical craniofacial morphology being lower in long-face subjects and higher in 
short-face subjects ${ }^{15}$. Previous studies ${ }^{12,17}$ have shown a strong positive correlation between masseter muscle thickness and maximal EMG activity. Additionally, interactions among MOF magnitude, jaw muscle size and craniofacial morphology have been demonstrated ${ }^{11,18}$. However, there is no agreement with regard to the role of the vertical facial craniofacial apparatus and muscular function $^{8,11}$.

Muscular forces exerted by the masticatory muscles can influence the success of prosthetic rehabilitation. The action of jaw muscles can generate interocclusal forces responsible for elastic flexure of the mandible and significant clinical modification of posterior sections of the lower arch during stomatognathic functioning ${ }^{14}$. Muscular contraction without tooth contact results in narrowing of the arch during opening and protrusion, and an increase during mandibular retrusion ${ }^{10,27,30}$. In contrast, during tooth contact the mandible can twist due to eversion of the masseteric processes, causing medial displacement of the coronoid processes ${ }^{13,30}$. As a consequence, the occurrence of these mandibular deformations can compromise the viability of long-term conventional fixed or implant-supported prostheses ${ }^{14}$.

Several attempts have been made to investigate the relationship between features of the mandibular muscles and the vertical facial pattern ${ }^{21}$. However, an integrated analysis of the jaw muscles and their effects on functional response with regard to craniofacial morphology has never been conducted in a homogeneous population. Additionally, previous studies $^{22,23}$ have evaluated the maximal occlusal force using thicker sensor pads, which lead to a greater distance between antagonistic teeth. The results may therefore not be reliable. Thus, this blinded controlled study aimed to evaluate the effects of different facial patterns on the maximal occlusion force, jaw muscle EMG activity during maximum voluntary clenches and the narrowing of the dental arch (MMF) in a sample of fully dentate adults.

\section{MATERIAL AND METHODS}

\section{Subjects}

The convenience sample of this cross-sectional study included patients and students recruited from Piracicaba Dental School, State University of Campinas, Brazil. The sample included 78 subjects (39 men and 39 women) with a mean age of 23.5 years (standard deviation: 4.0 ). All of them were healthy and without facial deformities. They had complete dentition (except for a lack of third molars) and showed no malocclusion, no occlusal vertical dimension alteration, no history of maxillofacial surgery or mandibular injuries, no orthodontic treatment for at least 2 years prior to the study, no periodontal disease, no caries, no temporomandibular disorders and parafunctional habits. The study protocol was approved by the Local Research and Ethics Committee, and all subjects signed written, informed consent. All subjects were submitted to anthropometric measurements, in which height was measured in centimeters $(\mathrm{cm})$ with the subject in the erect position without shoes and weight was recorded in kilograms $(\mathrm{kg})$ (Mechanical anthropometric scale R110; Welmy, Santa Bárbara D’Oeste, SP, Brazil).

\section{Vertical facial type evaluation}

Lateral cephalograms were used for facial type determination. The subjects were covered with a lead apron and placed in a cephalostat at a 90 degree angle to the tube head. All cephalograms were taken via standard procedures with the same radiographic unit (Elipsopantomograph Funk X-15; Macrotec Indústria e Comércio de Equipamentos Ltda., São Paulo, SP, Brazil) and processed with an automatic processor (Macrotec MX-2; Macrotec Indústria e Comércio de Equipamentos Ltda.). Facial patterns were determined by using digital cephalometric analysis (Radiocef v.4.0, Radio Memory Ltda, Belo Horizonte, MG, Brazil), which enabled skeletal type definition of each subject in the vertical direction. The vertical facial pattern was determined by the VERT index ${ }^{19}(\mathrm{VI})$, which is the arithmetic mean of the difference among the five cephalometric measures, facial axis (Ba-Na to Pt-Gn), facial depth ( $\mathrm{FH}$ to N-pog), mandibular height (FH to Go-Me), lower face height (ANS-Xi to Xi-Pm), and mandibular arch (Pm-Xi to Xi-Dc), divided by the standard deviation. The facial pattern of each subject was classified as (1) brachyfacial (VI above +0.5), (2) mesofacial (VI between -0.49 and +0.49 ) or (3) dolichofacial (VI below -0.5). Each group included 26 subjects.

\section{Maximum occlusal force measurement}

Bilateral maximum occlusal force was determined using a bite force sensor based on force-sensing resistors (FSR no. 151 NF; Interlink Electronics Inc., Camarillo, California, USA) ${ }^{9}$. The bite force sensors had a diameter of $12.7 \mathrm{~mm}$ and a thickness of $0.25 \mathrm{~mm}$. They were protected from moisture and deformities during clenching using metal disks of $0.7 \mathrm{~mm}$ in thickness, held by a plastic film. Thus, the total thickness of the sensor assembly was 2.25 $\mathrm{mm}$. Bite force sensor calibration was carried out by Instron 4411 (Tensile Test Machine, Tool Works Inc., Chicago, IL, USA). During the calibration procedure, compressive forces were applied by a lodged steel ball with a diameter of $3 \mathrm{~mm}$ to the sensor assembly at a loading range of 0-500 N. The force applied to the sensors was amplified, converted and recorded 
by analytical equipment (Spider 8; Hottinger Baldwin Messtechnik GmbH, Darmstadt, Hessen, Germany). The uses, limitations and reliability of these sensors have been previously discussed and reported ${ }^{9}$. Detailed experimental instructions were given to subjects prior to the actual recording session and a preliminary trial was made to build confidence in biting the force sensor as strong as possible in the test procedure. Each subject performed one maximum clench in the intercuspal position with the occlusion force sensors placed bilaterally in the first molar region, parallel to the occlusal plane, for $7 \mathrm{~s}$. The MOF value $(\mathrm{N})$ describes the maximum bilateral occlusion force of each subject and is defined as the sum of the maximum values from both sides ${ }^{28,29}$.

\section{Electromyographic examination}

The muscular activity of the masticatory muscles was recorded using a BioEMG electromyographic amplifier (BioEMG, Bioresearch Inc., Milwaukee, WI, USA), and the BioPAC software program (BioPAC Systems Inc., Santa Barbara, CA, USA). The 4-channel amplifier had a bandwidth of $30-600 \mathrm{~Hz}$, an input impedance $>100 \mathrm{M} \Omega$ and an input range of $1550 \mu \mathrm{VPP}$. Analogue-to-digital conversion was performed at a sampling rate of 1000 samples s-1 channel-1 with a maximal resolution of $0.625 \mu \mathrm{V}$ bit-1. In order to eliminate noise due to extraneous or cardiac artifacts, the program includes an additional $26 \mathrm{~dB}$ of $60 \mathrm{~Hz}$ noise reduction, reducing by $95 \%$ any record line-frequency interference in the EMG signals. Electromyographic activity was recorded from masseter and the anterior belly of temporal muscles bilaterally using surface bipolar, self-adhesive and pre-gelled electrodes with a contact area of $10 \mathrm{~mm}$ (BioPAC Systems Inc.). Skin impedance was reduced by alcohol scrub, and the electrodes were placed along the main direction of the muscle fibers, corresponding to the most voluminous part of the muscle, as determined during maximum contraction, $25 \mathrm{~mm}$ apart. The bipolar electrode configuration was used in every case, with a ground electrode placed near the $1^{\text {st }}$ thoracic vertebra ${ }^{3}$.

Data were recorded with the patients seated in a dental chair with the Frankfort horizontal plane parallel to the ground. Electromyographic activity of left and right masseter and temporal muscles was recorded during maximum voluntary clench on a film with a thickness of 0.020 " (ParafilmM; Structure Probe Inc., Menasha, WI, USA). Subjects were asked to clench the teeth in the intercuspal position as tightly as possible. During the endurance test, acoustic feedback on EMG activity level was provided to the subject in order to maintain isometric contraction of the masticatory muscles. Three recording tasks were performed during the same session, on each subject, with a resting period of $2 \mathrm{~min}$ after each evaluation. Seven seconds of EMG activity per task were recorded for each channel (muscle), but only the median interval of $5 \mathrm{~s}(71 \%)$ was used to quantify the EMG signal. In addition, EMG data were normalized by the maximum voluntary contraction of reference (MVRC), resulting in a percentage of the maximum reference contraction based on estimated marginal means. Maximum voluntary contraction for each muscle was calculated as the mean of three maximal clenches in the intercuspal position (5-second duration). The bilaterally measured EMG activity was the sum of the data obtained from the right and left masseter as well as the right and left temporalis ${ }^{29}$.

\section{Medial mandibular flexure analyses}

Impressions of the occlusal and incisal regions of the mandibular first molars of each subject were obtained by using a bite fork (George Gauge; Great Lakes Orthodontics Ltd., Tonawanda, NY, USA) as a tray for the vinyl polysiloxane putty material (Flexitime; Heraeus Kulzer GmbH, Hanau, Hessen, Germany). Impressions of each subject's occlusion were carried out in three mandibular positions: relative rest position (minimum mouth opening for impression procedure), maximum opening and maximum protrusion ${ }^{23}$. After the clinical session, the impressions obtained and a digital calipers with the measuring head set at $10-\mathrm{mm}$-width were scanned in a standardized fashion (HP Scanjet $6100 \mathrm{C} / \mathrm{T}$; Hewlett Packard Co, Houston, TX, USA) at fashion at $200 \%$ magnification and 300 dpi resolution. The images were analyzed to select two anatomical reference points located at the occlusal surface of the contralateral first molars. Analysis was performed by one previously calibrated observer. These reference points were recorded using the Adobe Photoshop 4.0 software tools (Adobe Systems Inc., San Jose, CA, USA). The width of the dental arch in each mandibular position was measured considering the intermolar linear distance set between these anatomical reference points by using the Image Tool software (University of Texas Health Science Center, San Antonio, TX, USA). The same procedure was carried out three times and a mean width of the dental arch in each position was obtained. MMF in maximum opening (MMFO) and protrusion (MMFP) were calculated by subtracting the width of the respective arch from the value obtained at the resting position ${ }^{22,23}$.

\section{Statistical analysis}

The comparison of MOF, masseter and temporal EMG activity and MMF in both positions among groups was assessed by one-way ANOVA. The normality of error distribution and the degree 
of non-constant variance were checked for each response variable using the SAS/LAB package (SAS software 8.01, SAS Institute Inc., Cary, NC, USA) and data were transformed according to Box, et al. ${ }^{2}$ (1978). Masseter EMG activity data were transformed to the inverse (1/Ra) and temporal EMG data was exponentially transformed; MOF and MMFO data were transformed by a logarithmic function $(\log 10)$ and MMFP to square root. PostANOVA comparisons were performed using Tukey HSD test. SAS software was used for all analysis and the significance limit was set at $5 \%$.

The errors of measurement (Se) for the bite force magnitude, EMG activity and the medial mandibular flexure measurements were also performed on repeated measurements ( $\mathrm{m} 1$, m2) of 10 randomly selected participants $(n)$, according to Dahlberg's $\mathrm{s}^{6}$ method: $\mathrm{Se}=\sqrt{ } \Sigma(\mathrm{m} 1$ $\mathrm{m} 2)^{2} / 2 \mathrm{n}$. Percentage error was calculated using the formula: $\%=(\mathrm{Se} / \mathrm{mean}) \times 100 \%$, where Se is the result from Dahlberg's ${ }^{6}$ (1940) formula and mean corresponds to the mean value of the initial and second measurements.

\section{RESULTS}

Anthropometric and sample characteristics are shown in Table 1 . No statistically significant differences were detected among the subjects' facial types when anthropometric measurements were considered $(P>0.05)$.

Means and standard deviations of MOF, masticatory muscle EMG activity and MMF are presented in Table 2. One-way ANOVA showed that there was a significant statistical difference $(P<0.0001)$ among all groups for MOF and masseter EMG activity during MVC $(P<0.05)$. Considering the brachyfacial group, both the MOF and masseter EMG activity values were significantly higher than those of the mesofacial and dolichofacial groups, respectively. There was no significant difference $(P>0.05)$ in the temporal EMG activity of brachyfacial and mesofacial groups, however both presented higher values than the dolichofacial group $(P<0.0001)$.

Significant differences for MMFO and MMFP were found among facial patterns $(P<0.0001)$ (Table 2). Brachyfacial subjects showed higher values of MMFO, followed by mesofacial and dolichofacial groups. However, in the MMFP group only dolichofacial subjects differed from the other two groups $(P<0.0001)$, showing lower values of MMF.

Error measurement - Maximum occlusal force (MOF) measurement error was $37.7 \mathrm{~N}$, approximately $9.1 \%$ for two consecutive recordings with a period of $5 \mathrm{~min}$ between the measurements. EMG error was determined during one session by two sets of repeated recordings with a period of 5 min between the tasks. EMG data error was small for masseter muscle, not exceeding 0.69 $\mu \mathrm{V}(0.2 \%)$. For temporalis muscles it was found to be larger, up to $1.36 \mu \mathrm{V}(2.9 \%)$. The reliability of the MMF data was determined on two sets of measurements with an interval of 14 days. The error of the individual measurements was $0.1 \mathrm{~mm}$ for MMFA and $0.2 \mathrm{~mm}$ for MMFP (approximately $5.3 \%$ and $4.9 \%$, respectively).

Table 1- Anthropometric characteristics of subjects (mean \pm standard deviation)

\begin{tabular}{|c|c|c|c|c|}
\hline \multicolumn{5}{|c|}{ Facial Pattern } \\
\hline & Brachyfacial & Mesofacial & Dolichofacial & Total \\
\hline & (VERT>+0.5) & $(-0.5 \leq$ VERT $\leq+0.5)$ & $($ VERT< $<-0.5)$ & \\
\hline Men (n) & 13 & 13 & 13 & 39 \\
\hline Age (years) & $23.9 \pm 4.5$ & $23.5 \pm 2.9$ & $22.9 \pm 3.8$ & $23.5 \pm 3.7$ \\
\hline Height (m) & $1.78 \pm 0.04$ & $1.75 \pm 0.06$ & $1.74 \pm 0.07$ & $1.75 \pm 0.06$ \\
\hline Weight (kg) & $75.6 \pm 9.9$ & $79.1 \pm 11.8$ & $75.0 \pm 10.3$ & $76.6 \pm 0.6$ \\
\hline Women (n) & 13 & 13 & 13 & 39 \\
\hline Age (years) & $23.4 \pm 4.2$ & $25.2 \pm 5.7$ & $22.1 \pm 2.2$ & $23.6 \pm 4.4$ \\
\hline Height (m) & $1.70 \pm 0.04$ & $1.66 \pm 0.06$ & $1.65 \pm 0.08$ & $1.67 \pm 0.06$ \\
\hline Weight (kg) & $63.1 \pm 5.4$ & $61.0 \pm 11.8$ & $59.2 \pm 7.4$ & $61.1 \pm 4.4$ \\
\hline Total (n) & 26 & 26 & 26 & 78 \\
\hline Age (years) & $23.7 \pm 4.3$ & $24.4 \pm 4.5$ & $22.5 \pm 3.1$ & $23.5 \pm 4.0$ \\
\hline Height (m) & $1.74 \pm 0.06$ & $1.70 \pm 0.07$ & $1.69 \pm 0.08$ & $1.71 \pm 0.07$ \\
\hline Weight (kg) & $69.4 \pm 10.1$ & $70.1 \pm 14.8$ & $67.1 \pm 11.9$ & $68.9 \pm 12.3$ \\
\hline
\end{tabular}


Table 2- Maximum occlusal force (MOF), combined (left and right) normalized electromyographic (EMG) activity for the masticatory muscles during maximum bilateral clenching (sEMG) and medial mandibular flexure in opening (MMFO) and protrusion (MMFP) positions, according to facial patterns (mean \pm standard deviation)

\begin{tabular}{lccc}
\hline & Brachyfacial & Facial Pattern & Dosofichofacial \\
\hline MOF $(\mathrm{N})^{*}$ & $524.5 \pm 153.0^{\mathrm{a}}$ & $389.7 \pm 162.8^{\mathrm{b}}$ & $272.6 \pm 149.1^{\mathrm{c}}$ \\
\hline $\mathrm{EMG}$ activity $(\% \mu \mathrm{V})$ & & & \\
Masseter * $^{*}$ & $76.0 \pm 5.4^{\mathrm{a}}$ & $75.2 \pm 5.6^{\mathrm{b}}$ & $75.0 \pm 3.6^{\mathrm{c}}$ \\
Temporal $^{*}$ & $85.1 \pm 4.7^{\mathrm{a}}$ & $85.0 \pm 9.2^{\mathrm{a}}$ & $84.7 \pm 2.9^{\mathrm{b}}$ \\
MMFO $(\mathrm{mm})^{*}$ & $0.30 \pm 0.15^{\mathrm{a}}$ & $0.21 \pm 0.13^{\mathrm{b}}$ & $0.14 \pm 0.08^{\mathrm{c}}$ \\
MMFP $(\mathrm{mm})^{*}$ & $0.23 \pm 0.09^{\mathrm{a}}$ & $0.19 \pm 0.12^{\mathrm{a}}$ & $0.09 \pm 0.07^{\mathrm{b}}$ \\
\hline
\end{tabular}

Different lower case letters show statistical differences among facial patterns. *Tukey test $(P<0.05)$

\section{DISCUSSION}

The research hypothesis was that maximum occlusion force, jaw muscle activity and medial mandibular flexure could be affected by vertical facial morphology. Previous reports have shown the influence of particular craniomorphologic characteristics on muscular activity patterns ${ }^{11,18}$. However, the present study assessed the effect of the overall vertical facial profile, by means of Ricketts analysis, on muscle force patterns and on medial mandibular flexure.

Sample characteristics may affect occlusal force production. Variables such as gender, age, height and weight, among several others, have the potential to modulate the generated occlusal force and pattern of muscle activity ${ }^{24}$. In this study, these variables were controlled (Table 1). Therefore, differences between subjects with distinct vertical facial patterns could be considered as a reliable representation of morphologic influence on muscular features ${ }^{22}$.

In this study, the EMG analysis allowed evaluating the activity of the masseter and temporal muscles groups at maximum effort condition. Besides, statistical differences did not predict differences in clinical terms, there were changes in the muscle activation pattern resulting from the vertical facial type. The fact that subjects with shorter face presented greater EMG activation, compared to long face subjects in the same clinical situations, can be a sign of muscular differences of the stomatognathic system caused by morphologic features of the craniomandibular complex, thus confirmed by the results of maximum occlusal force as demonstrated in the current research.

As assumed previously, the findings showed that both isolated EMG activity of the masticatory muscles and maximum occlusal force differed similarly according to facial type. All the evaluated muscular parameters showed that brachyfacial subjects exerted more effective muscular contraction than mesofacial and dolichofacial subjects. These results are in agreement with a previous study ${ }^{21}$ that observed a tendency of subjects with shorter vertical craniofacial apparatus to present higher bite force and higher levels of muscular activity during maximum clenching. A possible explanation for this is that craniomorphologic characteristics presented by brachyfacial subjects, such as lower gonial angle and minor maxillary height, can provide mechanical advantages to the stomatognathic system by forwarding the position of the load application point, which leads to a decrease in the loading moment arm, when compared to long-faced subjects ${ }^{26}$. Furthermore, it is well established that masseter and temporal muscles of brachyfacial subjects have larger cross-sectional areas ${ }^{12,13,17}$ and consequently greater muscular force ${ }^{18}$. This indicated that vertical facial morphology has a direct influence on muscular load generation.

In contrast, Shinkai, et al. ${ }^{22}$ (2007) reported no difference of MOF levels in subjects with different facial types. This may be due to methodological differences such as the sample and the thickness of the sensors used for MOF measurements. In the present study, the sample was homogeneous with regard to anthropometric characteristics and the number of volunteers in each group. In addition, the sensor assembly thickness used was around $2.25 \mathrm{~mm}$, inducing a smaller mouth opening and resulting in reduced displacement of the mandibular condyle on the articular eminence of the temporomandibular joint. This condition makes the evaluation of MOF more reliable and closer to the maximal intercuspal position ${ }^{9}$.

A previous study ${ }^{7}$ showed that under functional load, the human mandible presents complex elastic biomechanical behavior resulting in a width reduction of the arch during protrusion, as a result of muscular contraction independent of tooth contacts. In the present study, MMF data showed significant differences among facial types for MMFO 
$(P<0.05)$, which may be associated to differences in muscular stretching and endurance ${ }^{4,5}$. As a consequence of muscular work, the presence of craniomorphologic characteristics related to higher muscular strength could improve the degree of MMF generation ${ }^{16}$, as shown by the present data. MMFP values demonstrated that only the dolichofacial group presented reduced values $(P<0.05)$, about 2.5 times inferior to those of brachyfacial subjects. The onset of lateral pterygoid activity was delayed in jaw opening as compared to jaw protrusion ${ }^{27}$. During protrusion, both lateral and medial pterygoids are active, which results in decreased transverse arch dimensions, due to their action on the ascending mandibular ramus ${ }^{27}$. Therefore, the medial pterygoid activity of dolichofacial subjects could be decreased similarly to the lower values observed for masseter and temporal muscles, while action of lateral pterygoid muscle seems to be shortened. Its muscular stiffness may be caused by the difficulty of performing the protrusion movement during opening movement.

From a clinical point of view, the temporary morphologic alteration caused by muscular action over the mandibular bone may generate prosthesis misfit and discomfort during masticatory function ${ }^{1,9}$. Therefore the vertical facial pattern should be taken into consideration, regarding its influence on the phenomenon of mandibular deformation, in the decision-making processes and during follow up of patients using mandibular prostheses.

The present study showed that in an adult healthy population there are wide variations in muscular functional responses according to different craniofacial morphologies. Additionally, vertical facial profile of the dolichofacial subjects presents large inter-individual and intra-individual variation in specific morphologic characteristics ${ }^{5}$, which may also have contributed to the results observed. Our observations may help to explain why large variations in bilateral molar occlusion force, masticatory EMG activity and medial mandibular flexure can be found in subjects with apparently similar oral conditions. Despite the reliable results found, the surface EMG analyses only of masseter and temporal muscles could be considered a limitation of this study; the contribution of other muscular groups such as medial and lateral pterygoids was not taken into consideration. Therefore, future studies are necessary in order to verify the possible physiologic mechanisms involved in this complex relationship between functional responses of the stomatognathic system and morphologic craniofacial factors.

\section{CONCLUSION}

Based on the methodology, sample and results obtained in the present study, it was concluded that maximum occlusal force, masticatory EMG activity and medial mandibular flexure vary in relation to vertical facial patterns.

\section{ACKNOWLEDGEMENTS}

The authors would like to thank CNPq - National Council for Scientific and Technological Development (Grant \#476385/2004-0) for the financial support.

\section{REFERENCES}

1- Abdel-Latif HH, Hobkirk JA, Kelleway JP. Functional mandibular deformation in edentulous subjects treated with dental implants. Int J Prosthodont. 2000;13:513-9.

2- Box GE, Hunter WG, Hunter JS. Statistics for experimenters: an introduction to design, data analysis, and model building. New York: John Wiley \& Sons; 1978. p. 334-6.

3- Castroflorio T, Farina D, Bottin A, Piancino MG, Bracco P, Merletti R. Surface EMG of jaw elevator muscles: effect of electrode location and inter-electrode distance. J Oral Rehabil. 2005;32:411-7.

4- Chan HJ, Woods M, Stellac D. Mandibular muscle morphology in children with different vertical facial patterns: a 3-dimensional computed tomography study. Am J Orthod Dentofacial Orthop. 2008;133:10.e1-13.

5- Chen DC, Lai YL, Chi LY, Lee SY. Contributing factors of mandibular deformation during mouth opening. J Dent. 2000;28:583-8.

6- Dahlberg G. Statistical methods for medical and biological students. New York: Interscience Publications; 1940.

7- El-Sheikh AM, Abdel-Latif HH, Howell PG, Hobkirk JA. Midline mandibular deformation during nonmasticatory functional movements in edentulous subjects with dental implants. Int J Oral Maxillofac Implants. 2007;22:243-8.

8- Farella M, Bakke M, Michelotti A, Rapuano A, Martina R. Masseter thickness, endurance and exercise-induced pain in subjects with different vertical craniofacial morphology. Eur J Oral Sci. 2003;111:183-8.

9- Fernandes CP, Glantz POJ, Svensson SA, Bergmark A. A novel sensor for bite force determinations. Dent Mater. 2003;19:118-26. 10- Fischman, B. The rotational aspect of mandibular flexure. J Prosthet Dent. 1990;64:483-5.

11- Garcia-Morales P, Buschang PH, Throckmorton GS, English JD. Maximum bite force, muscle efficiency and mechanical advantage in children with vertical growth patterns. Eur J Orthod. 2003;25:265-72.

12- Georgiakaki I, Tortopidis D, Garefis P, Kiliaridis S. Ultrasonographic thickness and electromyographic activity of masseter muscle of human females. J Oral Rehabil. 2007;34:1218.

13- Hannam AG, Wood WW. Relationships between the size and spatial morphology of human masseter and medial pterygoid muscles, the craniofacial skeleton, and jaw biomechanics. Am J Phys Anthropol. 1989;80:429-45.

14- Lobbezoo F, Van Der Zaag J, Naeije M. Bruxism: its multiple causes and its effects on dental implants - an update review. J Oral Rehabil. 2006;33:293-300.

15- Pepicelli A, Woods M, Briggs C. The mandibular muscles and their importance in orthodontics: a contemporary review. Am J Orthod Dentofacial Orthop. 2005;128:774-80.

16- Proffit WR, Fields HW, Nixon WL. Occlusal forces in normal and long-face adults. J Dent Res. 1983;62:566-71. 
17- Raadsheer MC, Kiliaridis S, Van Eijden TM, Van Ginkel FC, Prahl-Andersen B. Masseter muscle thickness in growing individuals and its relation to facial morphology. Arch Oral Biol. 1996;41:323-32.

18- Raadsheer MC, Van Eijden TM, Van Ginkel FC, Prahl-Andersen B. Contribution of jaw muscle size and craniofacial morphology to human bite force magnitude. J Dent Res. 1999;78:31-42.

19- Ricketts RM. Perspective in the clinical application of cephalometrics. The first fifty years. Angle Orthod. 1981;51:11550.

20- Rowlerson A, Raoul G, Daniel Y, Close J, Maurage CA, Ferri J, et al. Fiber-type differences in masseter muscle associated with different facial morphologies. Am J Orthod Dentofacial Orthop. 2005; $127: 37-46$.

21- Serrao G, Sforza C, Dellavia C, Antinori M, Ferrario VF. Relation between vertical facial morphology and jaw muscle activity in healthy young men. Prog Orthod. 2003;4:45-51.

22- Shinkai RS, Canabarro SA, Schmidt CB, Sartori EA. Reliability of a digital image method for measuring medial mandibular flexure in dentate subjects. J Appl Oral Sci. 2004;12:358-62.

23- Shinkai RS, Lazzari FL, Canabarro SA, Gomes M, Grossi ML, Hirakata LM, et al. Maximum occlusal force and medial mandibular flexure in relation to vertical facial pattern: a cross-sectional study. Head Face Med. 2007;3:18.
24- Sondang P, Kumagai H, Tanaka E, Ozaki H, Nikawa H, Tanne $\mathrm{K}$, et al. Correlation between maximum bite force and craniofacial morphology of young adults in Indonesia. J Oral Rehabil. 2003;30:1109-17.

25- Sonnensen L, Bakke M. Molar bite force in relation to occlusion, craniofacial dimensions and head posture in pre-orthodontic children. Eur J Orthod. 2005;27:58-63.

26- Throckmorton GS, Finn RA, Bell WH. Biomechanics of differences in lower facial height. Am J Orthod. 1980;77:410-20. 27- Ueda R. The activity pattern of the inferior head of lateral pterygoid muscle. J Jpn Prosthet Soc. 1992;36:94.

28- Van Den Braber W, Van Der Glas H, Van Der Bilt A, Bosman F. Masticatory function in retrognathic patients, before and after mandibular advancement surgery. J Oral Maxillofac Surg. 2004;62:549-54.

29- Van Der Bilt A, Tekamp FA, Van Der Glas HW, Abbink J. Bite force and electromyography during maximum unilateral and bilateral clenching. Eur J Oral Sci. 2008;116:217-22.

30- Zarone F, Apicella A, Nicolais L, Aversa R, Sorrentino R. Mandibular flexure and stress build-up in mandibular full-arch fixed prostheses supported by osseointegrated implants. Clin Oral Implants Res. 2003;14:103-14. 